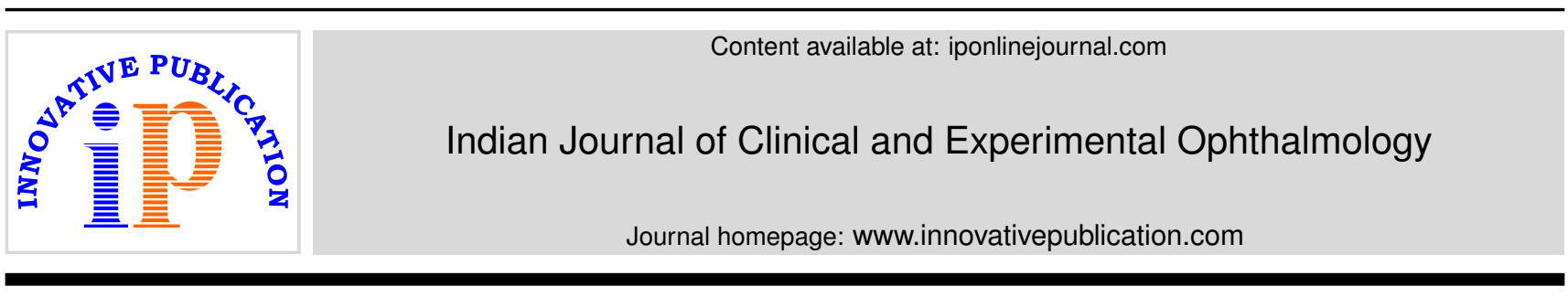

\title{
Editorial
}

\section{Traumatic glaucoma: An overview}

\author{
Rajendra Prakash Maurya ${ }^{1, *}$ \\ ${ }^{1}$ Regional Institute of Ophthalmology, Institute of Medical Sciences, Banaras Hindu University, Varanasi, Uttar Pradesh, India
}

A R T I C L E I N F O

Article history:

Received 11-03-2020

Accepted 12-03-2020

Available online 17-03-2020
(C) 2020 Published by Innovative Publication. This is an open access article under the CC BY-NC-ND license (https://creativecommons.org/licenses/by/4.0/)

\section{Dear Friends}

\section{Season's Greeting!!}

It is my humble privilege to present the special issue of IJCEO after successful journey of five years. This issue of IJCEO has several interesting articles on vision threatening morbidity, Glaucoma particularly on diagnosis and management of primary glaucoma.

Traumatic glaucoma (TG) refers to a heterogeneous group of post-traumatic secondary glaucoma with varying underlying mechanisms. Traumatic glaucoma occurs commonly after mechanical injury of globe (blunt or penetrating injury). Other conditions of traumatic glaucoma include post chemical, thermal, electric and radiation injury. The risk of traumatic glaucoma after a penetrating injury is approximately $2.67 \%$ while it is $3.39 \%$ after a blunt injury. ${ }^{1}$ Traumatic glaucoma can be open angle type (e.g. aqueous outflow obstruction by hyphaema/ghost cell) or angle closure variety (e.g. adherence of peripheral iris to the trabecular mesh work or peripheral cornea). Mechanism of TG may be anterior (pull) mechanism where abnormal tissue in angle; contracts and pulls the iris into the angle or posterior (push) mechanism (e.g. pupillary block by swollen traumatic cataract and forward movement of lens-iris diaphragm in case of cilio-choroidal effusion). Common causes of early onset TG in case

\footnotetext{
* Corresponding author.

E-mail addresses: editorijceo@gmail.com (R. P. Maurya).
}

of blunt trauma are (i) Trabecular meshwork disruption, (ii) Hyphaema: Accumulation of blood in the anterior chamber leads to obstruction of the trabecular meshwork with erythrocytes, fibrin, platelets and other inflammatory debris lead to prolonged increase in the IOP. $^{2}$ (iii) Inflammation: Trauma may also lead to direct inflammation of the trabecular meshwork (trabeculitis) causing decrease in aqueous outflow. (iv) Choroidal hemorrhage: causing a mass effect in the posterior segment leading to forward displacement of the lens-iris diaphragm and leads to acute angle closure glaucoma. The pathophysiology of late onset TG are (i) Angle-Recession (tear which occurs between the longitudinal and circular layers of the ciliary muscles) can cause raised IOP from 1 to 10 years after trauma. In gonioscopy scarred band can be seen. (ii) Ghost cell Glaucoma: Ghost cells are spherical, rigid and non flexible RBC occurring 3 weeks after trauma which clog the trabecular meshwork leading to increased IOP. ${ }^{3}$ (iii) Lens position: anterior dislocation / subluxation of the lens leading to pupillary block and acute angle closure glaucoma. (iv) Phacolytic Glaucoma: leakage of proteins through an intact lens capsule can cause severe inflammatory response in the trabecular meshwork and acute trabecular blockage. ${ }^{4}$ Rarely late TG can occur as Hemolytic Glaucoma (haemolytic products of RBCs can obstruct the trabecular meshwork) and Hemosiderotic Glaucoma (due to toxic effects of iron on the trabecular meshwork in case of long standing vitreous haemorrhage and retained IOFB). 
All mechanisms that can cause raise IOP after blunt trauma can also cause elevated IOP in case of penetrating injury. Hydrated lens can cause anterior bowing of iris and angle closure. TG is more common after alkali burn.

Management of post traumatic glaucoma is aimed mainly at the main underlying mechanism leading to increased IOP. The elevated IOP remains the main risk factor for glaucomatous optic neuropathy. The goal of treatment of hyphaema are to prevent the rebleed \& complications like corneal staining and to control IOP Topical beta blockers, topical carbonic anhydrase inhibitors, and alpha agonists are first line of drug and Prostaglandin analogues and miotics should be avoided. Oral acetazolamide and I.V Mannitol may also be used but avoided in patients with sickle cell disease. Surgical intervention in the form of anterior chamber washout indicated in case of persistant high IOP \& unresolving hyphema. Trabeculectomy might be required in case of trabecular meshwork disruption. Pars plana vitrectomy is recommended for Ghost cell glaucoma with dense vitreous haemorrhage not responding to medical treatment. Trabeculectomy with anti-metabolite and tube shunt surgery can be performed to control the IOP in case of angle-recession. ${ }^{5}$

\section{References}

1. Yanoff M, Duker JS, Jay S. Ophthalmology, Fifth Edition. vol. 2019. Philadelphia, Pennsylvania: Elsevier Inc ; 2019,.
2. Brandt MT, Haug RH. Traumatic hyphema: A comprehensive review. J Oral Maxillofac Surg. 2001;59(12):1462-1470. doi:10.1053/joms.2001.28284.

3. Alamri A, Alkatan H, Ibrahim A. Traumatic Ghost Cell Glaucoma with Successful Resolution of Corneal Blood Staining Following Pars Plana Vitrectomy. Middle East Afr J Ophthalmol. 2016;23(3):271-273.

4. Dhingra D, Grover S, Kapatia G, Pandav SS, Kaushik S. Phacolytic glaucoma: A nearly forgotten entity. Eur J Ophthalmol. 2019:

5. Khouri AS, Yadgarov A, Liu D, Crane ES. Surgical Outcomes of Ahmed or Baerveldt Tube Shunt Implantation for medically Uncontrolled Traumatic Glaucoma. J Curr Glaucoma Pract. 2017;11(1):16-21.

\section{Author biography}

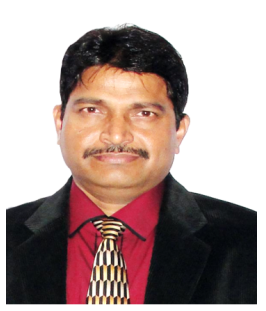

Rajendra Prakash Maurya (MS, Ph.D) Editor in Chief IJCEO, Senior Assistant Professor \& I/c Ocular Oncology, Trauma and Oculoplasty

Cite this article: Maurya RP. Traumatic glaucoma: An overview.

Indian J Clin Exp Ophthalmol 2020;6(1):1-2. 